\title{
Halofuginone induces the apoptosis of breast cancer cells and inhibits migration via downregulation of matrix metalloproteinase-9
}

\author{
MEI LING JIN ${ }^{2 *}$, SUN YOUNG PARK ${ }^{1 *}$, YOUNG HUN KIM ${ }^{1}$, GEUNTAE PARK $^{3}$ and SANG JOON LEE ${ }^{2}$ \\ ${ }^{1}$ Bio-IT Fusion Technology Research Institute, ${ }^{2}$ Department of Microbiology, ${ }^{3}$ Institute for Research \\ and Industry Cooperation, Pusan National University, Busan 609-735, Republic of Korea
}

Received September 2, 2013; Accepted October 14, 2013

DOI: $10.3892 /$ ijo.2013.2157

\begin{abstract}
Halofuginone (HF) is extracted from Dichroa febrifuga, a plant used in traditional medicine. We report that the HF extract inhibits the growth of breast cancer cells and induces the generation of reactive oxygen species (ROS) and apoptosis, an important feature of potential anticancer agents. In addition, $\mathrm{HF}$ significantly reduces the migration and invasion of MCF-7 and MDA-MB-231 human breast cancer cells after 12-O-tetraecanoylphorbol-13-acetate (TPA) stimulation. As matrix metalloproteinase-9 plays a critical role in tumor metastasis, we analyzed its expression with the HF extract treatment. Western blot analysis and gelatin zymography showed that HF suppresses MMP-9 expression and activity concentrationdependently. HF also decreases the nuclear protein levels of nuclear factor kappa B (NF- $\mathrm{BB})$ and c-fos (AP-1), critical transcription factors regulating MMP-9 expression through binding the MMP-9 promoter region. Luciferase assays showed that HF decreases TPA-induced MMP-9 promoter binding activities of NF- $\kappa$ B and AP-1. Taken together, these are the first results indicating that halofuginone may represent a promising new agent for breast cancer chemotherapy.
\end{abstract}

\section{Introduction}

Breast cancer is the predominant type of cancer among women and is the second leading cause of cancer-related mortality (1). Similar to other solid tumors, breast cancer is difficult to treat. Some traditional methods, such as chemotherapy, may cause

Correspondence to: Professor Sang-Joon Lee, Department of Microbiology, Pusan National University, Busan 609-735, Republic of Korea

E-mail: sangjoon@pusan.ac.kr

Dr Geuntae Park, Institute for Research and Industry Cooperation, Pusan National University, Busan 609-735, Republic of Korea E-mail: gtpark@pusan.ac.kr

*Contributed equally

Key words: Halofuginone, ROS, apoptosis, invasion, MMP-9 strong side effects and drug resistance in patients. Therefore, there is continuing need to find novel, efficient and less toxic cancer therapeutic molecules. Natural products, such as plant-derived drugs, have played an increasing role in cancer treatment due to their fewer side effects and high efficacy.

The small molecule Halofuginone (HF) is an analog of quinazolinone alkaloid, a derivative of febrifugine originally isolated from the plant Dichroa febrifuga. It has been used as a drug, receiving certification from the US Food and Drug Administration in the early 1980s and is used for preventing coccidiosis infection in growing chickens and turkeys (2). In vitro, $\mathrm{HF}$ was first reported to inhibit collagen type I synthesis and extracellular formation via the reduction of matrix metalloproteinase-2 (MMP-2) (3). Halofuginone has also been shown to inhibit cancer cell growth and tumor metastasis in cell lines derived from hepatoma (4), melanoma (5) and multiple myeloma (6). In vivo, oral treatment with $\mathrm{HF}$ (at concentrations of 0.1-0.4 $\mathrm{mg} / \mathrm{kg}$ per day) significantly reduced brain tumor growth and angiogenesis in a metastatic brain tumor model in rats (7). This study focused on the effect of HF on breast cancer based on its potent effects on various other types of tumor growth and metastasis.

Recently methods have been developed to prove the drug effectiveness as an anticancer compound. One of these strategies is to determine the ability and mechanism of novel anticancer agents to induce apoptosis in cancer cells. Therefore, various apoptotic pathways have been extensively investigated for many compounds to understand their cytotoxicity. A sensitive effector mechanism widely engaged in the control and modulation of apoptosis is cellular redox status, which is determined by the balance between the rates of generation and breakdown of reactive oxygen species (ROS) (8). ROS is a major regulator involved in apoptosis mediated through mitochondria. High levels of ROS in mitochondrial can induce several irreversible steps. First, free radicals may attack membrane phospholipids, causing mitochondrial membrane depolarization and the release of mitochondrial factors. This intrinsic apoptosis pathway is subsequently involved in the activation of caspases and DNA fragmentation (9).

Several novel anticancer agents extracted from plants, such as dryofragin (9), diallyl trisulfide (8) and levamisole (10) can inhibit various types of cancer cells by inducing apoptosis via 
the generation of ROS and the disruption of mitochondrial transmembrane potential. Centratherum anthelminticum (L.) seeds induce apoptosis in breast cancer MDA-MB-231 cells, which is associated with ROS generation and downregulation of the mitochondrial proteins Bcl2 and Bcl-xl (11).

New anticancer agents have also been reported to control various other processes involved in the malignant transformation of cells, such as invasion and metastasis (12). The invasive behavior of cancer cells and their ability to metastasize to a distant location are multiple step processes associated with the detachment of cells from the original cancer, attachment to extracellular matrix (ECM) binding sites, degradation of the ECM and migration to target tissues (13). One of the critical steps involved in metastasis is the activity of MMPs that degrade a variety of ECM proteins (14). Of these MMPs, both MMP-2 and MMP-9 are the key enzymes that control the rate of cell invasion and metastasis $(15,16)$. Therefore, the downregulation of MMP-2 and MMP-9 can be used as an important strategy for metastasis intervention and preventing cancer progression. Although these two gelatinases have similar properties, their gene expression is differentially and specifically regulated by distinct regulatory elements in their promoter regions (17). Compared to MMP-2, which is constitutively expressed, MMP-9 is strongly related to the malignant phenotype of various cancers and highly induced by a variety of stimuli, including epidermal growth factor (EGF), cytokines, UV radiation, oncogenes and 12- $O$-tetradecanoylphorbol-13-acetate (TPA) (16). Among these inducers, TPA can increase MMP-9 expression and secretion during breast cancer cell invasion through activating transcription factors including activator protein (AP-1) and nucleus factor kappa B (NF-kB), as its promoter region contains binding sites for these transcription factors (17).

Despite the diverse studies on the biological activities of $\mathrm{HF}$, the potential of HF against breast cancer growth and invasion is poorly defined. The goal of this study was to evaluate the ability of HF to inhibit tumor growth and metastasis in the human breast cancer lines, MCF-7 and MDA-MB-231 cells. We also investigated the underlying pathways involved in inhibiting breast cancer cell growth, migration and invasion by HF. Furthermore, we show that HF induces the apoptosis of breast cancer cells and inhibits their migration via downregulating MMP-9 expression.

\section{Materials and methods}

Reagents. BD BioCoat ${ }^{\mathrm{TM}}$ Matrigel $^{\mathrm{TM}}$ invasion chambers were obtained from BD Biosciences (San Jose, CA, USA). Cell culture media (RPMI-1640) and fetal bovine serum (FBS) were purchased from Gibco BRL (now part of Invitrogen Corp., Carlsbad, CA, USA). FuGENE-6 transfection regent was purchased from Roche Applied Science (Indianapolis, IN, USA). Antibodies against MMP-2, MMP-9, phosphorylated $\mathrm{p} 65, \mathrm{p} 65$, phosphorylated $\mathrm{I} \kappa \mathrm{B} \alpha, \mathrm{I} \kappa \mathrm{B} \alpha, \mathrm{c}$-fos and TBP were purchased from Cell Signaling Technology (Beverly, MA, USA). Halofuginone was purchased from Sigma-Aldrich (St. Louis, MO, USA).

Cell culture. Two human breast cancer cell lines, MCF-7 and MDA-MB-231 cells, were grown in RPMI supplemented with $10 \%$ fetal bovine serum, $100 \mathrm{U} / \mathrm{ml}$ penicillin and
$100 \mu \mathrm{g} / \mu 1$ streptomycin (Biochrom AG, Berlin, Germany). Cell cultures were grown to confluence and maintained in a humidified atmosphere at $37^{\circ} \mathrm{C}$ and $5 \% \mathrm{CO}_{2}$. The MCF-7 and MDA-MB-231 cells were incubated with fetal bovine serumfree medium for $24 \mathrm{~h}$ prior to the experiments, HF was then added to a final concentration in the range 1-200 $\mathrm{nM}$.

MTT cell viability assay. The cell viability of MCF-7 and MDA-MB-231 cells was evaluated using a microculture (3-(4,5-dimethylthiazol-2-yl)-2,5-diphenyltetrazolium bromide (MTT)-based colorimetric assay. Cells were incubated in 24-well plates at a density of $5 \times 10^{4}$ cells per well. MTT solution $(5 \mu \mathrm{lof} 5 \mathrm{mg} / \mathrm{ml})$ was added to each well (final concentration, $62.5 \mu \mathrm{g} / \mathrm{ml})$. After incubation for $4 \mathrm{~h}$ at $37^{\circ} \mathrm{C}$ in $5 \% \mathrm{CO}_{2}$, the supernatant was removed and the formazan crystals produced in the viable cells were solubilized in $150 \mu \mathrm{l}$ of dimethylsulfoxide. The absorbance of each well was then read at $570 \mathrm{~nm}$ using a microplate reader (Wallac 1420, Boston, MA, USA).

Measurement of intracellular ROS. To evaluate intracellular ROS levels, cells were treated with CM- $\mathrm{H}_{2}$ DCFDA (Invitrogen, Paisley, UK), an indicator of general oxidative stress, for $1 \mathrm{~h}$ at $37^{\circ} \mathrm{C}$ under $5 \% \mathrm{CO}_{2}$. ROS are capable of oxidizing cleaved DCFH to DCF. The cells were then harvested and washed three times with PBS. Fluorescence intensity was measured using flow cytometry with an excitation wavelength of $488 \mathrm{~nm}$ and an emission wavelength of $525 \mathrm{~nm}$. Data analyses were performed using CXP software 2.0 (Beckman Coulter, Brea, CA, USA).

Analysis of mitochondrial membrane potential $\left(\Delta \psi_{m}\right)$. The mitochondrial membrane potential $\left(\Delta \psi_{m}\right)$ of MCF-7 and MDA-MB-231 cells were determined by 5,5,6,6-tetrachloro-1,1,3,3-tetraethylbenzimidazolcarbocyanine iodide (JC-1) reduction. JC-1 is a sensitive indicator of $\Delta \psi_{m}$, which causes its fluorescence to change from red to green. MCF-7 and MDA-MB-231 cells were stained with JC-1 and analyzed by subsequent flow cytometry. JC-1 red florescence, indicating intact $\Delta \psi_{m}$, was excited at $488 \mathrm{~nm}$ and the emission detected using a $613 \pm 20-\mathrm{nm}$ band pass filter. For each sample, 10,000 cells were acquired and analyzed by flow cytometry. Data were analyzed using the fluorescence intensity of the analyzed cell population.

Cell cycle analysis and sub-G1 measurement. We plated $1 \times 10^{6}$ cells on $6-\mathrm{cm}$ dishes for $24 \mathrm{~h}$. The cells were incubated with $200 \mathrm{nM}$ HF for $24 \mathrm{~h}$, followed by the collection of cells by centrifugation. The pellets were mixed with $75 \%$ ethanol at $-20^{\circ} \mathrm{C}$ overnight. The cells were then centrifuged and resuspended in $300 \mu \mathrm{l}$ PBS containing $1 \%$ PI for $10 \mathrm{~min}$ at room temperature in the dark. The cells were immediately evaluated by flow cytometry. For each sample, 10,000 cells were acquired and analyzed by flow cytometry.

Annexin V/PI dye staining. Cells were seeded on 6-well plates at a density of $3 \times 10^{5}$ cells/well. After SS treatment $(50,100$ and $300 \mu \mathrm{g} / \mathrm{ml}$ ) for $72 \mathrm{~h}$, cells were stained with Annexin V and propidium iodide (Clontech, CA, USA) for $15 \mathrm{~min}$ at room temperature. The apoptotic index was determined by flow cytometry with FlowJo software. Triplicate independent experiments were performed. 
Terminal deoxynucleotidyl transferase dUTP nick-end labeling (TUNEL assay). The TUNEL assay is a common method for detecting DNA fragmentation that results from apoptotic signaling cascades. This assay relies on the presence of nicks in the DNA which can be identified by terminal deoxynucleotidyl transferase (TdT), an enzyme that will catalyze the addition of dUTPs that are secondarily labeled with a marker. TUNEL was performed on MCF-7 and MDA-MB-231 cells fixed for 10 min with $2 \%$ paraformaldehyde, washed twice in $0.1 \mathrm{M}$ Tris buffer, fixed again for $1 \mathrm{~min}$ in acetone, washed and incubated for $1 \mathrm{~h}$ at $37^{\circ} \mathrm{C}$ with $1.5 \mu \mathrm{M}$ fluorescein isothiocyanate-coupled dUTP (Boehringer-Mannheim) plus terminal deoxynucleotidyl transferase (TdT, $0.5 \mathrm{IU} / \mu \mathrm{l}$; Boehringer-Mannheim) in $1 \mathrm{X}$ TdT buffer. After two more washes the cells were analyzed by flow cytometry. For each sample, 10,000 cells were acquired and analyzed by flow cytometry. Data analyses were performed with CXP software 2.0 (Beckman Coulter).

Gelatin zymography. To assess MMP-9 and MMP-2 gelatinase activity, MCF-7 and MDA-MB-231 cells were grown in 6-well plates. The cells were washed once with serum-free medium and placed in serum-free medium for $24 \mathrm{~h}$ prior to HF. Conditioned media was collected and prepared with standard SDS-gel loading buffer containing 0.01\% SDS without $\beta$-mercaptoethanol and not boiled before loading. Prepared samples were then subjected to electrophoresis with $8 \%$ SDS polyacrylamide gels containing $0.1 \%$ gelatin. After electrophoresis, gels were wash twice with $150 \mathrm{ml} 2.5 \%$ Triton-X-100 for $20 \mathrm{~min}$ at room temperature (to remove SDS) and then incubated in $150 \mathrm{ml}$ incubation buffer $(50 \mathrm{mM}$ Tris- $\mathrm{HCl}, \mathrm{pH} 8.0$, $10 \mathrm{mM} \mathrm{CaCl}_{2}, 0.02 \% \mathrm{NaN}_{3}$ ) for $16 \mathrm{~h}$ at $37^{\circ} \mathrm{C}$, stained with Coomassie brilliant blue R-250 and destained with destaining solution (20\% methanol, $10 \%$ acetic acid and $70 \%$ water).

Cell invasion assay. The cell invasion assay was conducted using BD BioCoat ${ }^{\mathrm{TM}}$ Matrigel $^{\mathrm{TM}}$ invasion chambers (BD Biosciences) according to the manufacturer's instructions. Briefly, MDA-MB-231 cells $\left(5 \times 10^{4}\right)$ and MCF-7 cells $\left(5 \times 10^{4}\right)$ suspended in $500 \mu \mathrm{l}$ of serum-free medium in the presence or absence of HF were seeded into upper chamber of filter inserts coated with growth factor containing Matrigel. After $1 \mathrm{~h}, 500 \mu \mathrm{l}$ of serum-free medium containing $80 \mathrm{nM}$ TPA was added to the bottom wells. Chambers were incubated at $37^{\circ} \mathrm{C}$ for $24 \mathrm{~h}$, after which cells that had invaded the lower surface of the filter insert were methanol-fixed for $15 \mathrm{~min}$ and stained with $0.2 \%$ crystal violet for 15 min before being counted under the light microscope using a 10x objective.

Wound healing assay. To study the effects of HF on cell migration in vitro, a wound healing assay was performed using MCF-7 and MDA-MB-231 cells. These cells were seeded in 6 -well plates and grown until the plates were $90 \%$ confluent. Cells were then serum-starved for $12 \mathrm{~h}$. A linear wound was created in the confluent monolayer using a $200-\mathrm{ml}$ pipette tip. Cells were then observed using a phase contrast microscope.

Preparation of nuclear extract. MCF-7 and MDA-MB-231 cells $\left(3 \times 10^{6}\right)$ were washed three times with cold PBS and the cell pellets suspended in hypotonic buffer (Active Motif, Carlsbad, CA, USA) and incubated for $15 \mathrm{~min}$ on ice. Then
$250 \mu \mathrm{l}$ of detergent (Active Motif) was added to the cell extract and the sample was incubated on ice for $1 \mathrm{~min}$. The extract was centrifuged at $13,000 \mathrm{rpm}$ for $1 \mathrm{~min}$ at $4^{\circ} \mathrm{C}$. After removing the $10-\mu 1$ cytosolic protein-containing supernatant, nuclear proteins were extracted by adding complete $30 \mu \mathrm{l}$ lysis buffer B (Active Motif) for $30 \mathrm{~min}$ at $4^{\circ} \mathrm{C}$ with occasional vortexing. After centrifugation at $13,000 \mathrm{rpm}$ for $5 \mathrm{~min}$ at $4^{\circ} \mathrm{C}$, the $28 \mu \mathrm{l}$ supernatants were collected and stored at $-70^{\circ} \mathrm{C}$.

Western blot analysis. MCF-7 and MDA-MB-231 cells were harvested in ice-cold lysis buffer consisting of $1 \%$ Triton $\mathrm{X}-100,1 \%$ deoxycholate and $0.1 \%$ sodium dodecyl sulfate (SDS). Protein content in the cell lysates was determined using the Bradford assay (Bio-Rad, Hercules, CA, USA). Total protein in each sample $(50 \mu \mathrm{g})$ was resolved using 7.5\% SDS-polyacrylamide gel electrophoresis (SDS-PAGE), transferred to a polyvinylidene difluoride membrane and incubated with the appropriate antibodies. The proteins were visualized using an enhanced chemiluminescence detection system (Amersham Biosciences, Piscataway, NJ, USA) with horseradish peroxidase-conjugated anti-rabbit or anti-mouse secondary antibodies. Western blotting was used to determine the level of MMP-9 (1:1,000 dilution), MMP-2 (1:1,000 dilution), P-P65 (1:1,000 dilution), P65 (1:1,000 dilution), p-ІкB $\alpha$ (1:1,000 dilution), c-fos (1:1,000 dilution), TBP (1:1,000 dilution) and tubulin (1:5,000 dilution). Images were acquired using an ImageQuant 350 analyzer (Amersham Biosciences, Little Chalfont, UK).

Luciferase assay. To determine promoter activity, we used a dual-luciferase reporter assay system (Promega, Madison, WI, USA). MDA-MB-231 cells were transfected with an $\mathrm{NF}-\kappa \mathrm{B}$ luciferase reporter plasmid or AP-1 luciferase reporter plasmid (Stratagene, Grand Island, NY, USA) using FuGENE-6 reagent (Roche Applied Science) according to the manufacturer's instructions. Renilla luciferase control plasmid pRL-CMV (Promega) was co-transfected as an internal control of transfection efficiency. Cells were incubated with the indicated reagents for $1 \mathrm{~h}$ and then treated with TPA for $24 \mathrm{~h}$. Luciferase was assayed with a dual-luciferase assay kit (Promega) according to the manufacturer's instructions. Luminescence was measured with a GloMax ${ }^{\mathrm{TM}} 96$ microplate luminometer (Promega).

Statistical analysis. All results were expressed as the mean \pm SE. Each experiment was repeated at least three times. Statistical significance was measured between each treated group and analyzed by the paired Student's t-test. Data with $\mathrm{p}<0.05$ were considered statistically significant.

\section{Results}

HF is cytotoxic to breast cancer cells and induces the accumulation of intracellular ROS. To evaluate the effect of HF on the viability of breast cancer cells, MCF-7 and MDA-MB-231 cells were exposed to increasing concentrations of $\mathrm{HF}$ (from 25 to $800 \mathrm{nM}$ ) for 24,48 and $72 \mathrm{~h}$ and the percentage of viable cells were determined using the MTT assay. HF decreased cell viability in a time- and concentration-dependent manner in MCF-7 and MDA-MB-231 breast cancer cells (Fig. 1A and B). 
A

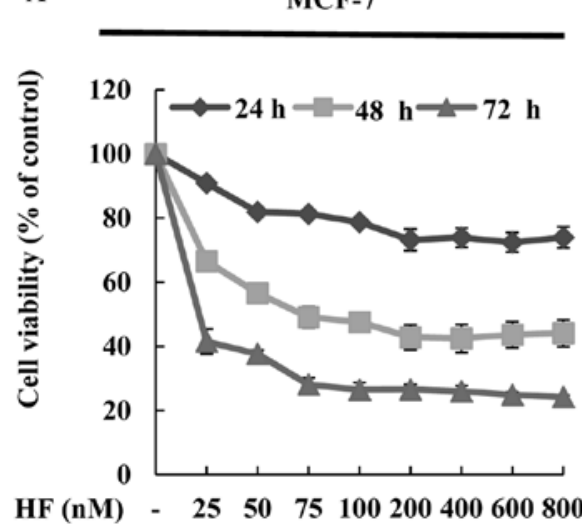

B

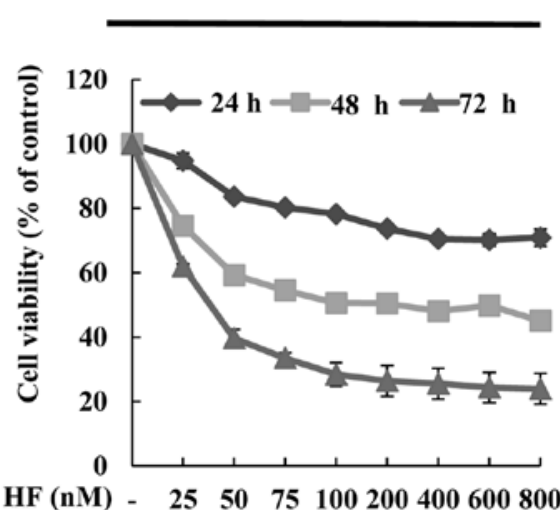

C

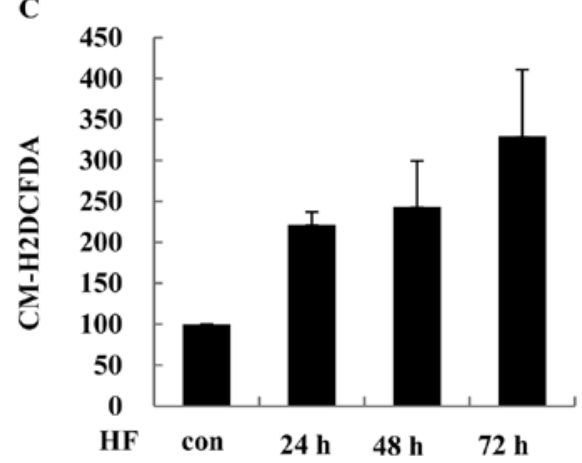

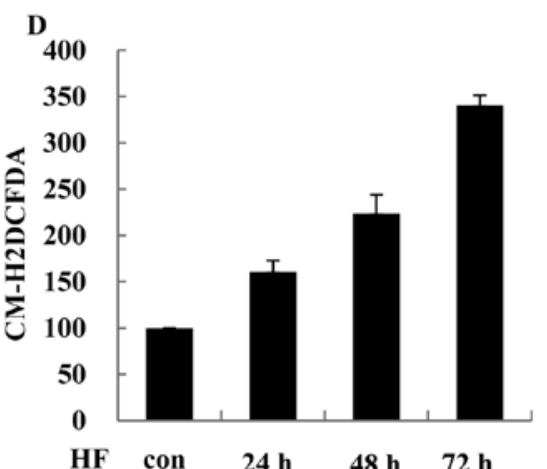

Figure 1. HF induces growth inhibition and ROS generation in breast cancer cells. (A and B) MCF-7 and MDA-MB-231 cells were treated with different concentrations of $\mathrm{HF}(25-800 \mathrm{nM})$ for 24, 48 and $72 \mathrm{~h}$. The inhibitory rate was determined by the MTT assay. (B) MCF-7 and MDA-MB-231 cells were treated with $200 \mathrm{nM} \mathrm{HF}$ for 24, 48 and $72 \mathrm{~h}$ and ROS levels measured by JC-staining. Stained cells were analysed by flow cytometry.

At $48 \mathrm{~h}$, the $\mathrm{IC}_{50}$ values of $\mathrm{HF}$ were found to be $200 \mathrm{nM}$ in MCF-7 cells and $200 \mathrm{nM}$ in MDA-MB-231 cells. Next, to determine whether intracellular ROS are involved in the cytotoxic effects of HF on these two cell lines, flow cytometry was used to detect changes in CM- $\mathrm{H}_{2}$ DCFDA fluorescence intensity in MCF-7 and MDA-MB-231 cells at 24, 48 and $72 \mathrm{~h}$. Treatment with HF increased the intracellular production of ROS in a time-dependent manner, indicating that HF induced the accumulation of intracellular ROS in MCF-7 cells and MDA-MB-231 cells (Fig. 1C and D).

$H F$ induces apoptotic cell death in breast cancer cells. To determine whether HF could induce apoptosis in breast cancer cells, cells were treated with $200 \mathrm{nM}$ HA for indicated times and the apoptotic cell percentage was quantified by flow cytometry. HF increased the sub-G1 percentage of cells for two breast cancer cell lines compared to the control (Fig. 2A and B). To confirm this result, we also performed the TUNEL assay to detect the apoptosis levels induced by HF. The results showed that the percentage of apoptotic cell death significantly increased in HF-treated breast cancer cells (Fig. 2C and D).

HF induces apoptosis and disrupts mitochondrial membrane potential in breast cancer cells. To further evaluate the effect of HF on the induction of apoptosis, both breast cancer cell lines were incubated with $200 \mathrm{nM} \mathrm{HF}$ and then double-stained with Annexin V-FITC and PI, followed by quantitative flow cytometry analysis. After treatment with HF for $72 \mathrm{~h}$, the cells presented features of early apoptosis in both cell lines (Fig. 3A and B). Compared to the control, the percentage of both early and late apoptotic cells increased with the addition of $200 \mathrm{nM}$ HF. Based on our results, ROS may play a critical role in HF-induced apoptosis in both breast cancer cell lines. The mitochondria are a major source for ROS generation and are involved in ROS-related apoptosis. Disruption of mitochondrial transmembrane potential is related to mitochondrial dysfunction, which is linked to apoptosis and the loss of cell viability. To analyze whether mitochondria are involved in HF-induced apoptosis, we measured MMP changes using the JC-1 stain. When breast cancer cells were exposed to HF, the mitochondrial membrane potential strongly increased. These results indicate that HF can induce the loss of viability and apoptosis through enhancing intracellular ROS oxidative stress in MCF-7 and MDA-MB-231 breast cancer cells.

HF inhibits the migration and invasion of breast cancer cells. Cancer cells migration and invasion are critical steps of tumor metastasis. We tested cell motility with HF treatment in the wound-healing assay. TPA strongly increased MCF-7 and MDA-MB-231 cell migration by $24 \mathrm{~h}$, but HF reduced migration levels by $24 \mathrm{~h}$ (Fig. 4A and B). In previous results, $\mathrm{HF}$ had inhibitory effects on the viability of breast cancer cells; therefore there was a possibility that the HF-mediated inhibition of invasion was a consequence of its cytotoxic effects. To 
A

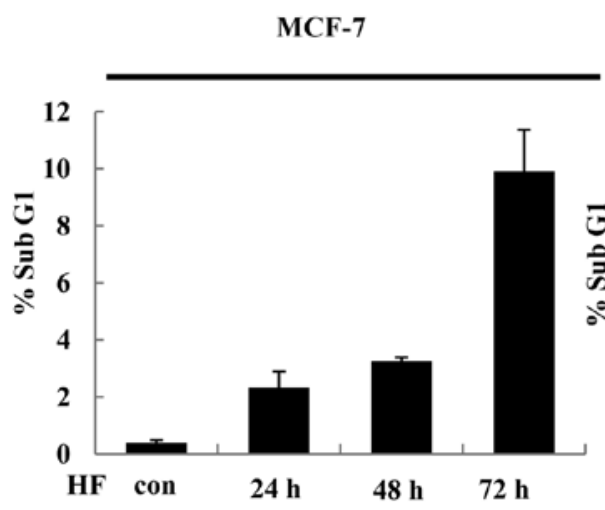

C

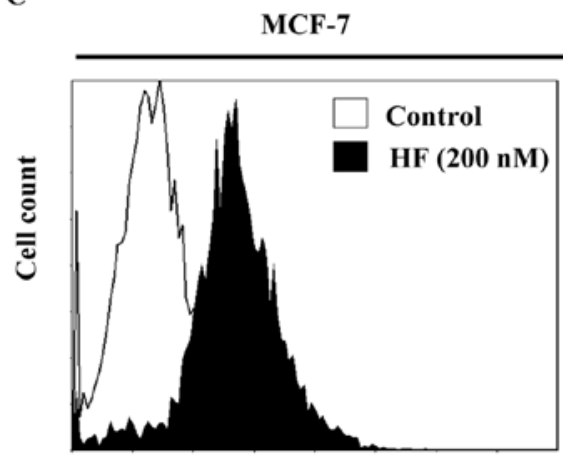

Fluorescence intensity

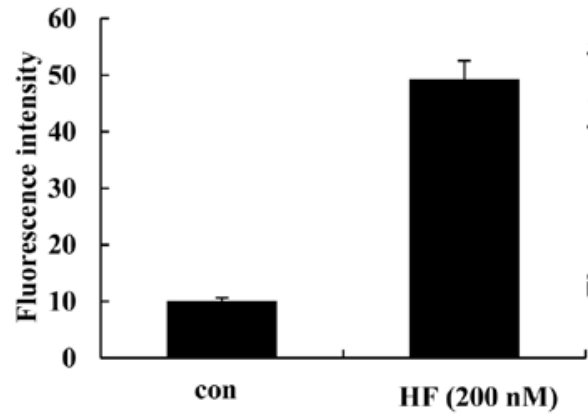

MDA-MB-231

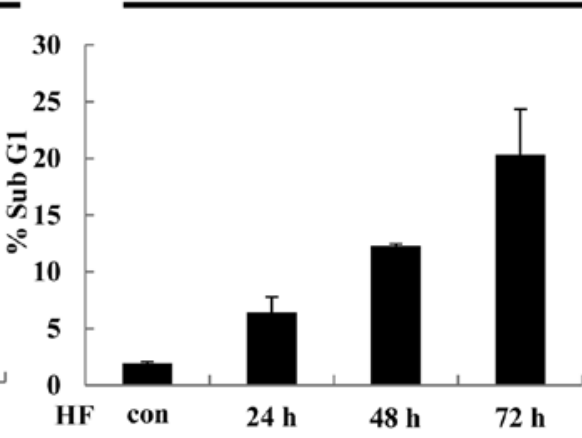

D

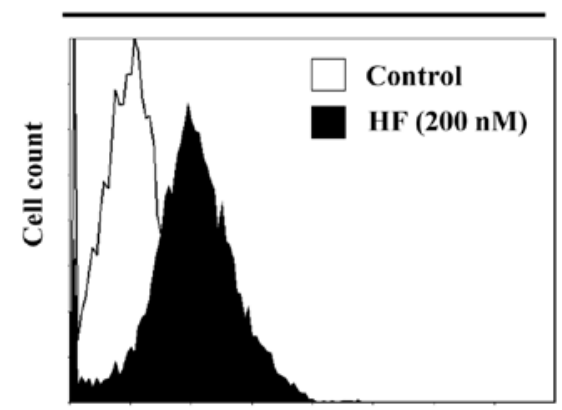

Fluorescence intensity

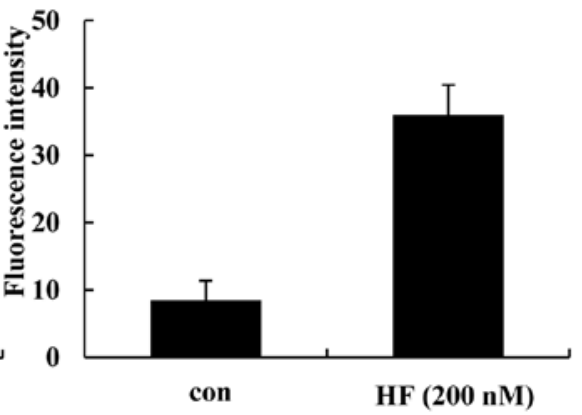

Figure 2. HF induces apoptosis in breast cancer cells. (A and B) MCF-7 and MDA-MB-231 cells were treated with $200 \mathrm{nM} \mathrm{HF}$ for 24, 48 and $72 \mathrm{~h}$. Apoptotic cell frequencies were calculated as the percentage of cells falling to the left of the G1 peak (sub-G1) of the total number of cells analyzed. Cell death was determined by propidium iodide binding followed by flow cytometry. (C and D) MCF-7 and MDA-MB-231 cells were treated with $200 \mathrm{nM} \mathrm{HF}$ for $72 \mathrm{~h}$ and the cells were subjected to the TUNEL assay. TUNEL-stained cells were quantified by flow cytometry. The number in each panel is the percentage of TUNELpositive cells. The results represent the findings of three independent experiments.

address this, we specifically examined the invasive behavior of breast cancer cell cultures in the presence or absence of HF in the transwell chamber assay. TPA induced cancer cell invasion (Fig. 4C and D); however, HF treatment decreased the invasive ability of both MCF-7 and MDA-MB-231 cancer cells. Taken together, our data suggest that HF inhibits the migration and invasion of MCF-7 and highly metastatic MDA-MB-231 cells in vitro.

HF decreases MMP-9 gene expression and activity. MMP-2 and MMP-9 are key proteins in the process of invasion and metastasis. Gelatin zymography was performed to investigate whether HF had inhibitory effects on TPA-induced MMP-2 and MMP-9 activity. Whereas MMP-2 activity remained unaffected, TPA-stimulated MMP-9 gelatinolytic activity was prevented by $\mathrm{HF}$ in both MCF-7 and MDA-MB-231 cancer cells (Fig. 5A and B). In agreement with the findings described above, HF treatment of the cells was found to decrease TPA-induced MMP-9 protein expression in both MCF-7 and MDA-MB-231 cancer cells (Fig. 5C and D).

$H F$ decreases $N F-\kappa B$ and AP-1 gene expression and nuclear translocation. The promoter region of the MMP-9 gene contains binding sites for transcription factors, including AP-1 and NF- $\kappa$ B. Whether the inhibitory effect of HF on MMP-9 expression can occur through NF- $\mathrm{B}$ and AP-1 activities was examined. Luciferase reporter assays performed on MCF-7 cells (transfected with NF- $\kappa$ B and AP-1 luciferase reporter plasmids) showed that TPA-enhanced $N F-\kappa B$ and AP-1 transcriptional activity was decreased by HA (Fig. 5F 
A

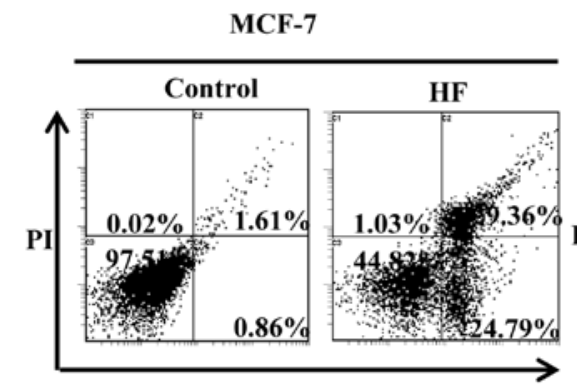

Annexin V

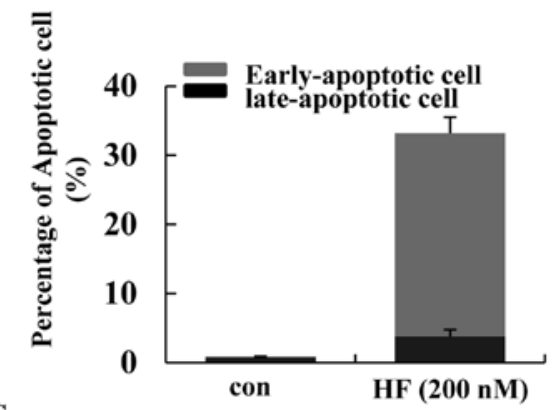

C

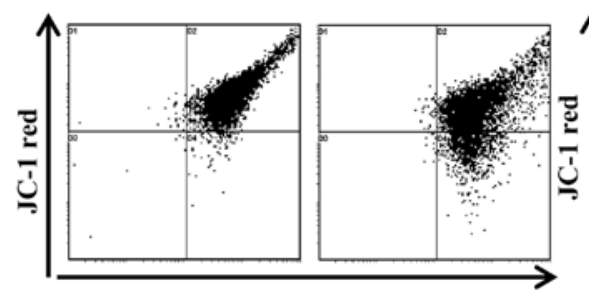

JC-1 green

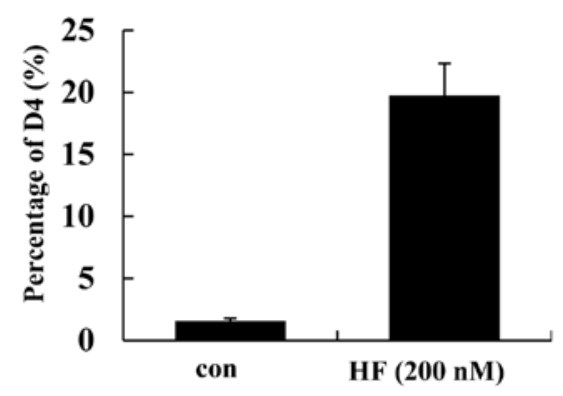

B

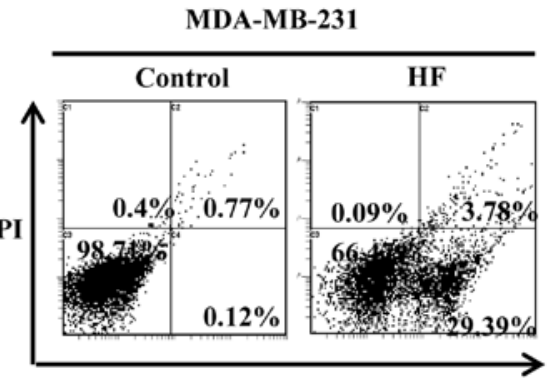

Annexin V
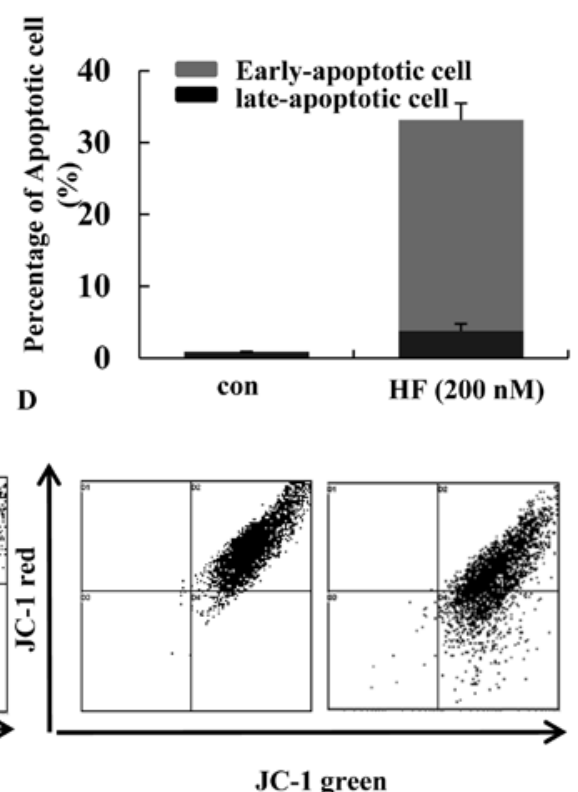

JC-1 green

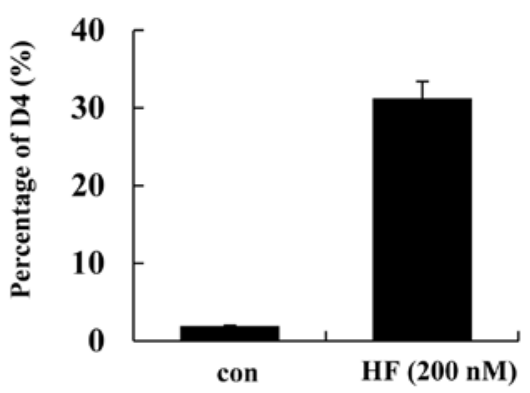

Figure 3. HF induces apoptosis and a disordered mitochondrial membrane potential (MMP, $\Delta \psi$ ). (A and B) MCF-7 and MDA-MB-231 cells were doublestained with Annexin V-FITC and PI and then cells were analyzed by flow cytometry. (C and D) Evaluation of mitochondria membrane potential in MCF-7 and MDA-MB-231 cells was done with JC-1 staining followed by flow cytometry. All experiments were done independently in triplicate per experimental point and representative results are shown.

and $\mathrm{G})$. NF- $\mathrm{NB}$ is normally sequestered in the cytoplasm in an inactive form associated with its inhibitory protein, $\mathrm{I} \kappa \mathrm{B} \alpha$. Upon activation, the $N F-\kappa B$ p65 subunit is released to the nucleus to initiate target gene transcription as a result of $I_{\kappa} \mathrm{B} \alpha$ degradation. AP-1 contains two subunits, c-fos and c-Jun, that can translocate to the nucleus when AP-1 is activated. In Western blot analysis, TPA increased the nuclear translocation of the NF- $\mathrm{B}$ subunit p-p65 and the AP-1 subunit c-fos. In addition, TPA-stimulated NF- $\kappa$ B p65 and AP-1 c-fos nuclear translocation were reduced by HF treatment (Fig. 5E). In addition, HF was found to increase the TPA-induced reduction of cytoplasmic p-p65. Taken together, these results suggest that the inhibitory effect of $\mathrm{HF}$ on $\mathrm{NF}-\kappa \mathrm{B}$ and AP-1 signaling path- ways can be attributed to its inhibition of p65 and c-fos nuclear translocation and the binding activity of transcription factors.

\section{Discussion}

Epidemiological studies have revealed an inverse association between the dietary intake of natural compounds and cancer risk $(11,19)$. Various types of natural agents extracted from plants, including alkaloid compounds with anticancer activity, have recently been identified $(20,21)$. One promising therapeutic candidate is Halofuginone, an orally available synthetic derivative of a quinazolinone alkaloid that has shown preclinical antitumor activity in various models, including human 
A

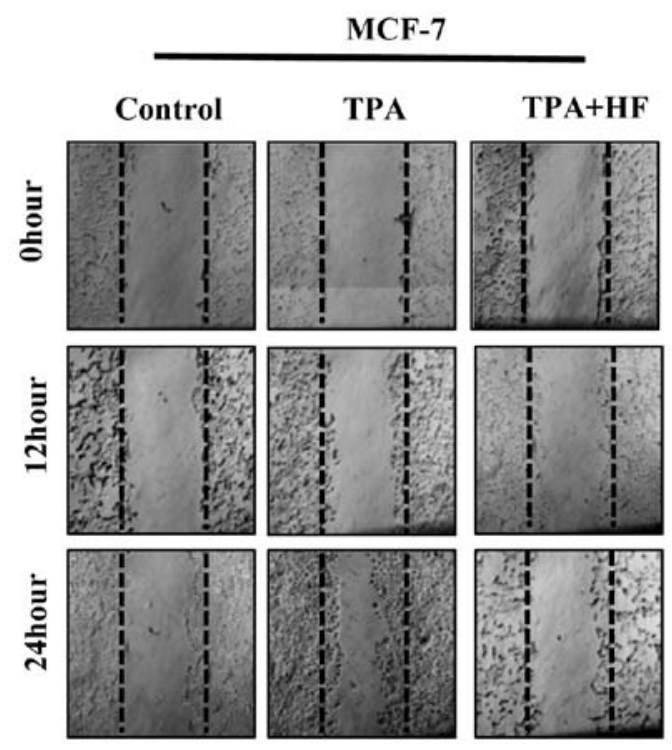

C
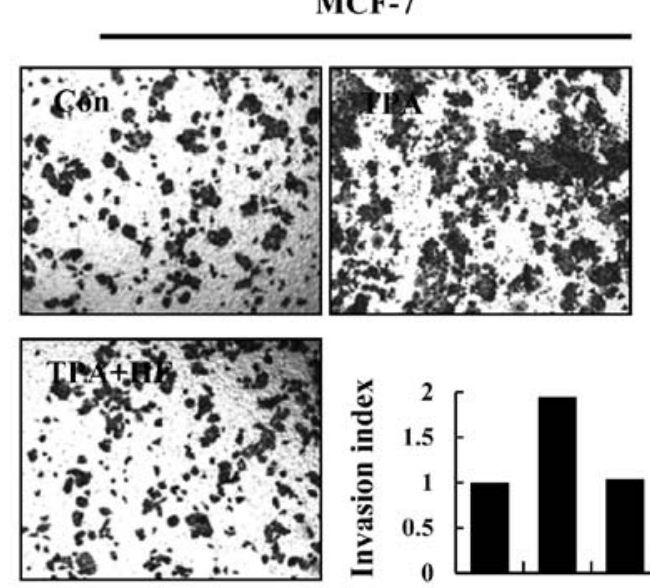

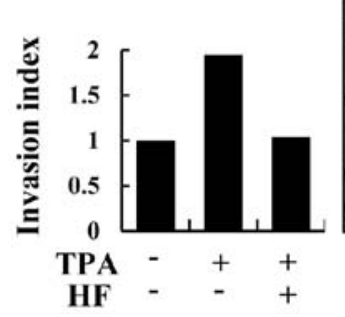

B

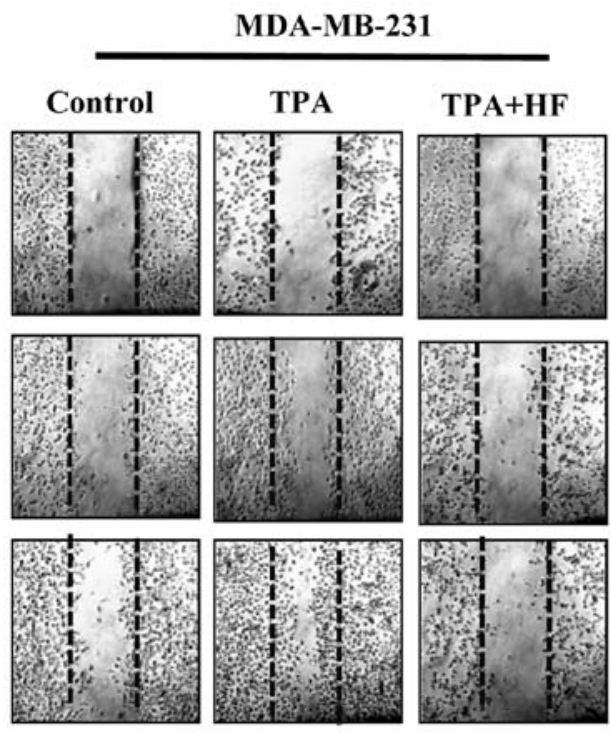

D

MDA-MB-231
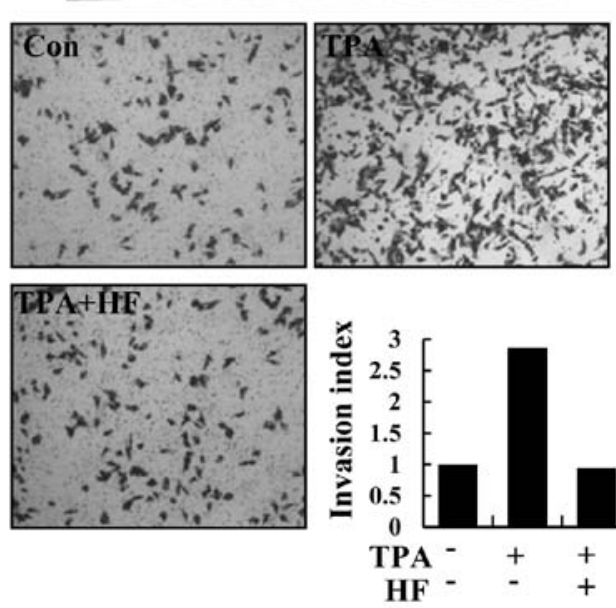

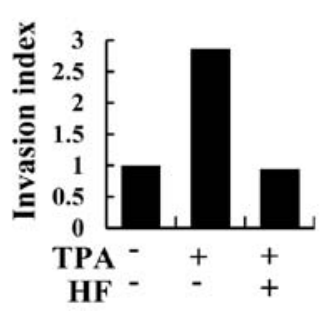

Figure 4. HF affects TPA-stimulated migration and invasion in breast cancer cells. (A and B) The serum-starved cell monolayer was scratched with a pipette tip, pretreated with or without $200 \mathrm{nM} \mathrm{HF}$ for $1 \mathrm{~h}$, then $200 \mathrm{nM}$ TPA applied for $24 \mathrm{~h}$. The results were observed under phase contrast microscope and photographed. (C and D) MCF-7 and MDA-MB-231 cells were treated with $200 \mathrm{nM}$ HF for $24 \mathrm{~h}$ and the invasion ability of cells was quantified by counting the number of cells that invaded the underside of the porous polycarbonate membrane under a microscope.

melanoma bone metastases $(18,22)$. In the present study, we assessed the anticancer effects of HF and the mechanism of these effects.

The capability of anticancer drugs to induce the cellular apoptosis of cancer cells is an important property of cancer treatment $(23,24)$. We observed a time-dependent apoptotic pattern with $\mathrm{HF}$ exposure that was similar between MCF-7 and MDA-MB-231 (Fig. 2A). Further evidence emerged from TUNEL assay results, which determined a strong elevation in apoptotic rate for both cell lines (Fig. 2).

In addition, we confirmed that $\mathrm{HF}$ induced apoptosis by causing the accumulation of ROS levels. ROS inducers as anticancer drugs have received considerable attention due to their selective effect on cancer cells while sparing normal cells (25). Emerging evidence suggests that ROS induced apoptotic cell death in cancer cells by causing mitochondrial membrane damage, resulting in a mitochondrial membrane potential disorder (26-28). We examined the mitochondrial membrane potential using a JC-1 stain and our results suggest that $\mathrm{HF}$ induces ROS generation and affects mitochondrial dynamics, inducing the dysfunction of mitochondrial membrane potential (Fig. 3B).

We observed an inhibitory effect of HF on TPA-induced migration and invasion in MCF-7 and MDA-MB-231 breast cancer cell lines. Accumulating evidence suggests that MMP-2 and MMP-9 expression are strongly implicated in breast cancer cell invasion (29). Effective anticancer agents involved in anti-invasion have demonstrated the ability to downregulate MMP-2 or MMP-9 expression. Our data reveal that HF inhibits TPA-induced MMP-9 expression, but does not influence MMP-2 expression levels in either MCF-7 or MDA-MB-231 breast cancer cells (Fig. 5). TPA treatment 
A

MCF-7

B

MDA-MB-231
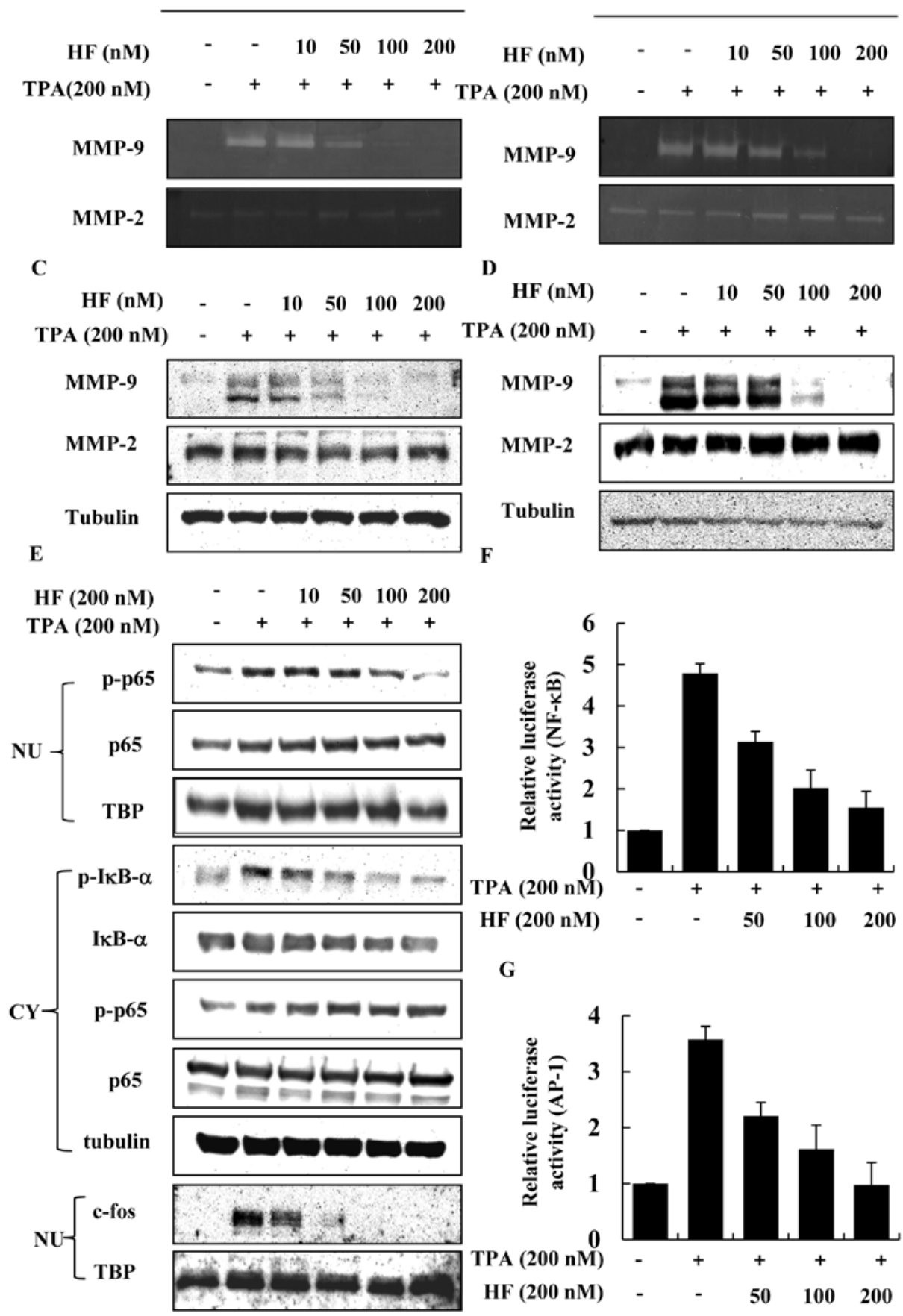

Figure 5. HF inhibits the activities and expression levels of MMPs and MMP-9 upstream transcription factor NF-кB and AP-1 in breast cancer cells. MCF-7 and MDA-MB-231 cells were treated with various concentrations of $\mathrm{HF}(10,50,100$ and $200 \mathrm{nM})$ for $1 \mathrm{~h}$ and then treated with $200 \mathrm{nM}$ TPA and subjected to (A and B) gelatin zymography to analyze the activities of MMP-2 and MMP-9. Conditioned media were collected and activity of MMP-2/MMP-9 was determined. (C and D) Western blot analysis of MMP-2 and MMP-9 protein levels. Tubulin served as an internal control of protein levels. (E) MCF-7 cells were treated with various concentrations of HF for $1 \mathrm{~h}$ and following $200 \mathrm{nM}$ TPA treatment, nuclear extracts (p-p65, p65 and c-fos) and cytosolic extracts (p-IкB $\alpha$, IкB $\alpha, p-p 65$ and p65) were subjected to SDS-PAGE, followed by western blot analysis. TBP and tubulin served as nuclear and cytosolic internal controls, respectively. Proteins were visualized with the ECL detection system. (F and G) Cells were treated with various concentrations of HF for $1 \mathrm{~h}$ followed by $200 \mathrm{nM}$ TPA treatment, then the luciferase assay was performed to detect the promoter activity of NF- $\mathrm{kB}$ and AP-1 in MCF-7 cells.

strongly increased MMP-9 gelatinolytic activity, but did not similarly increase MMP-2 gelatinolytic activity. The result was consistent with a previous observation that only MMP-9 is induced with TPA and that MMP-9 plays a central role in the TPA-induced invasion of MCF-7 cells (16). MMP-9 regulates tumor progression and invasion on two levels, including activity and expression (30). MMP-9 is a critical enzyme required for degrading type IV collagen, a major component of the basement membrane. According to previous reports, inflammatory cytokines (IFN- $\gamma$ ), growth factors (HGF, EGF) and TPA stimulate MMP-9 by activating distinct intracellular signaling pathways in breast cancer cells $(31,32)$. The 
mechanism of MMP-9 expression induced by TPA is thus important for the development of a therapeutic experimental model of tumor metastasis.

To reveal the mechanisms of the TPA-induced signaling cascade involved in MMP-9 expression in MCF-7 cells, we investigated the effects of two transcription factors, NF- $\kappa \mathrm{B}$ and AP-1 and their binding activity. It is well-known that MMP-9 expression can be influenced by AP-1 and NF- $\kappa \mathrm{B}$ binding at its promoter region. The MMP-9 promoter contains DNA-binding sites for these transcription factors; thus, many differences in the promoter of these two MMPs could create different responses to TPA between the two MMPs (31). Therefore, the specific responsiveness of MMP-9 demonstrated that its downregulation by HF was changed through an upstream event. In agreement, we showed that HF reduced TPA-induced NF- $\kappa$ B and AP-1 transcriptional activity and translocation. These findings indicate that the reduction of MMP-9 and its transcription factors by HF might contribute to the prevention of TPA-induced invasive growth.

AP-1, which belongs to the bZIP group of DNA binding proteins, is comprised of homodimers or heterodimers via a combination of signaling events. This leads to the increasing activity of proteins that can directly regulate c-jun or c-fos expression (35). On the other hand, the transcription factor $\mathrm{NF}-\kappa \mathrm{B}$ forms a family of inducible transcription factors that regulates numerous genes associated with inflammation, cancer development and tumor invasion.

Accumulating reports have suggested that many biologically active natural phytochemicals such as curcumin, resveratrol and isoliquiritigenin suppress NF- $\mathrm{BB}$ and AP-1 expression in cancer cells and eventually prevent cancer cell invasion $(16,33,34)$. Exposure of cells to specific stimuli, including TPA, results in IKK complex activation, which in turn targets $\mathrm{I} \kappa \mathrm{B} \alpha$ phosphorylation and degradation by the ubiquitin-proteasome machinery. This causes the NF- $\mathrm{BB}$ p65 subunit to translocate to the nucleus and bind the MMP-9 promoter to initiate MMP-9 gene transcription (36). In our results, HF reduced TPA-induced NF- $\kappa \mathrm{B}$ p65 and AP-1 c-fos nuclear translocation through preventing I $\kappa \mathrm{B} \alpha$ phosphorylation. The NF- $\kappa \mathrm{B}$ and AP-1 elements are centrally implicated in TPA-induced MMP-9 gene induction at two levels, including nuclear translocation and transcription activity. Therefore the inhibition of MMP-9 expression and its upstream elements can be a critical strategy involved in treating malignant tumors, including breast cancer.

In conclusion, the present study demonstrates that the natural compound HF exhibits effective antitumor properties. The observed antitumor activity inhibits the growth of cancer cells and induces apoptosis. In addition, our study provides evidence that HF can inhibit the migration and invasion of breast cancer cells through the downregulation of MMP-9 expression and its upstream transcription factors. Moreover, we revealed that the molecular mechanism by which $\mathrm{HF}$ inhibits MMP-9 expression is mediated through its suppressive effect on NF- $\kappa \mathrm{B}$ and AP-1 activation. As tumor metastasis is often associated with poor prognosis and high mortality among breast cancer patients, the need to discover and develop new therapeutic strategies that target early tumor invasiveness or metastasis. In this regard, $\mathrm{HF}$ is a promising agent against breast cancer invasion and metastasis.

\section{Acknowledgements}

This study was supported by the Basic Science Research Program through the National Research Foundation of Korea (NRF) funded by the Ministry of Education, Science and Technology (2012R1A1A3010601).

\section{References}

1. Liao YF, Rao YK and Tzeng YM: Aqueous extract of anisomeles indica and its purified compound exerts anti-metastatic activity through inhibition of NF-kappaB/AP-1-dependent MMP-9 activation in human breast cancer MCF-7 cells. Food Chem Toxicol 8: 2930-2936, 2012.

2. de Jonge MJ, Dumez H, Verweij J, et al: Phase I and pharmacokinetic study of halofuginone, an oral quinazolinone derivative in patients with advanced solid tumours. Eur J Cancer 12: 1768-1774, 2006.

3. Zcharia E, Atzmon R, Nagler A, Shimoni A, Peretz T, Vlodavsky I and Nagler A: Inhibition of matrix metalloproteinase- 2 by halofuginone is mediated by the Egr1 transcription factor. Anticancer Drugs 10: 1022-1031, 2012.

4. Nagler A, Ohana M, Shibolet O, et al: Suppression of hepatocellular carcinoma growth in mice by the alkaloid coccidiostat halofuginone. Eur J Cancer 9: 1397-1403, 2004.

5. van Kempen LC, Rijntjes J, Mamor-Cornelissen I, et al: Type I collagen expression contributes to angiogenesis and the development of deeply invasive cutaneous melanoma. Int J Cancer 5: 1019-1029, 2008.

6. Leiba M, Jakubikova J, Klippel S, et al: Halofuginone inhibits multiple myeloma growth in vitro and in vivo and enhances cytotoxicity of conventional and novel agents. Br J Haematol 6: 718-731, 2012.

7. Abramovitch R, Itzik A, Harel H, Nagler A, Vlodavsky I and Siegal T: Halofuginone inhibits angiogenesis and growth in implanted metastatic rat brain tumor model - an MRI study. Neoplasia 5: 480-489, 2004.

8. Na HK, Kim EH, Choi MA, Park JM, Kim DH and Surh YJ: Diallyl trisulfide induces apoptosis in human breast cancer cells through ROS-mediated activation of JNK and AP-1. Biochem Pharmacol 10: 1241-1250, 2012.

9. Zhang Y, Luo M, Zu Y, et al: Dryofragin, a phloroglucinol derivative, induces apoptosis in human breast cancer MCF-7 cells through ROS-mediated mitochondrial pathway. Chem Biol Interact 2: 129-136, 2012.

10. Hegde M, Karki SS, Thomas E, et al: Novel levamisole derivative induces extrinsic pathway of apoptosis in cancer cells and inhibits tumor progression in mice. PLoS One 9: e43632, 2012.

11. Looi CY, Arya A, Cheah FK, et al: Induction of apoptosis in human breast cancer cells via caspase pathway by vernodalin isolated from centratherum anthelminticum (L.) seeds. PLoS One 2: e56643, 2013.

12. Li F, Li C, Zhang H, et al: VI-14, a novel flavonoid derivative, inhibits migration and invasion of human breast cancer cells. Toxicol Appl Pharmacol 2: 217-226, 2012.

13. Artacho-Cordon F, Rios-Arrabal S, Lara PC, Artacho-Cordon A, Calvente I and Nunez MI: Matrix metalloproteinases: potential therapy to prevent the development of second malignancies after breast radiotherapy. Surg Oncol 3: 143-151, 2012.

14. Yu HY, Kim KS, Moon HI, Kim KM, Lee YC and Lee JH: JNP3, a new compound, suppresses PMA-induced tumor cell invasion via NF-kappaB down regulation in MCF-7 breast cancer cells. Biochem Biophys Res Commun 2: 190-196, 2012.

15. Jin J, Cai L, Liu ZM and Zhou XS: miRNA-218 inhibits osteosarcoma cell migration and invasion by down-regulating of TIAM1, MMP2 and MMP9. Asian Pac J Cancer Prev 6: 3681-3684, 2013.

16. Kim JM, Noh EM, Kwon KB, et al: Curcumin suppresses the TPA-induced invasion through inhibition of PKCalphadependent MMP-expression in MCF-7 human breast cancer cells. Phytomedicine 12: 1085-1092, 2012.

17. Ling H, Zhang Y, Ng KY and Chew EH: Pachymic acid impairs breast cancer cell invasion by suppressing nuclear factor-kappaBdependent matrix metalloproteinase- 9 expression. Breast Cancer Res Treat 3: 609-620, 2011.

18. Juarez P, Mohammad KS, Yin JJ, et al: Halofuginone inhibits the establishment and progression of melanoma bone metastases. Cancer Res 23: 6247-6256, 2012. 
19. Spector I, Zilberstein Y, Lavy A, Nagler A, Genin O and Pines M: Involvement of host stroma cells and tissue fibrosis in pancreatic tumor development in transgenic mice. PLoS One 7: e41833, 2012.

20. Bournine L, Bensalem S, Peixoto P, et al: Revealing the antitumoral effect of algerian glaucium flavum roots against human cancer cells. Phytomedicine 13: 1-8, 2013.

21. Liu JM, Pan F, Li L, et al: Piperlongumine selectively kills glioblastoma multiforme cells via reactive oxygen species accumulation dependent JNK and p38 activation. Biochem Biophys Res Commun 1: 87-93, 2013.

22. Grudzien MM, Low PS, Manning PC, Arredondo M and Belton RJ Jr and Nowak RA: The antifibrotic drug halofuginone inhibits proliferation and collagen production by human leiomyoma and myometrial smooth muscle cells. Fertil Steril 4: 1290-1298, 2010.

23. Chandra-Kuntal K, Lee J and Singh SV: Critical role for reactive oxygen species in apoptosis induction and cell migration inhibition by diallyl trisulfide, a cancer chemopreventive component of garlic. Breast Cancer Res Treat 138: 69-79, 2013.

24. Shi X, Zhao Y, Jiao Y, Shi T and Yang X: ROS-dependent mitochondria molecular mechanisms underlying antitumor activity of pleurotus abalonus acidic polysaccharides in human breast cancer MCF-7 cells. PLoS One 5: e64266, 2013.

25. Huang P, Feng L, Oldham EA, Keating MJ and Plunkett W: Superoxide dismutase as a target for the selective killing of cancer cells. Nature 6802: 390-395, 2000.

26. Petanidis S, Kioseoglou E, Hadzopoulou-Cladaras M and Salifoglou A: Novel ternary vanadium-betaine-peroxido species suppresses $\mathrm{H}$-ras and matrix metalloproteinase-2 expression by increasing reactive oxygen species-mediated apoptosis in cancer cells. Cancer Lett 2: 387-396, 2013.

27. Yang L, Wang $\mathrm{P}$, Wang $\mathrm{H}$, et al: Fucoidan derived from undaria pinnatifida induces apoptosis in human hepatocellular carcinoma SMMC-7721 cells via the ROS-mediated mitochondrial pathway. Mar Drugs 6: 1961-1976, 2013.

28. Yang F, Chen WD, Deng R, et al: Hirsutanol A, a novel sesquiterpene compound from fungus Chondrostereum sp., induces apoptosis and inhibits tumor growth through mitochondrialindependent ROS production: hirsutanol A inhibits tumor growth through ROS production. J Transl Med 32: doi:10.1186/14795876-11-32, 2013.
29. Chen HW, Chao CY, Lin LL, Lu CY, Liu KL, Lii CK and Li CC: Inhibition of matrix metalloproteinase-9 expression by docosahexaenoic acid mediated by heme oxygenase 1 in 12-O-tetradecanoylphorbol-13-acetate-induced MCF-7 human breast cancer cells. Arch Toxicol 87: 857-869, 2013.

30. Lee WJ, Chen WK, Wang CJ, Lin WL and Tseng TH: Apigenin inhibits HGF-promoted invasive growth and metastasis involving blocking PI3K/Akt pathway and beta 4 integrin function in MDA-MB-231 breast cancer cells. Toxicol Appl Pharmacol 2: 178-191, 2008.

31. Chao CY, Lii CK, Hsu YT, Lu CY, Liu KL, Li CC and Chen HW: Induction of heme oxygenase-1 and inhibition of TPA-induced matrix metalloproteinase-9 expression by andrographolide in MCF-7 human breast cancer cells. Carcinogenesis 34: 1843-1851, 2013.

32. Ma L, Lan F, Zheng Z, et al: Epidermal growth factor (EGF) and interleukin (IL)-1beta synergistically promote ERK1/2-mediated invasive breast ductal cancer cell migration and invasion. Mol Cancer 11: doi: 10.1186/1476-4598-11-79, 2012.

33. Liu PL, Tsai JR, Charles AL, et al: Resveratrol inhibits human lung adenocarcinoma cell metastasis by suppressing heme oxygenase 1-mediated nuclear factor-kappaB pathway and subsequently downregulating expression of matrix metalloproteinases. Mol Nutr Food Res 54: 196-204, 2010

34. Li Y, Zhao H, Wang Y, et al: Isoliquiritigenin induces growth inhibition and apoptosis through downregulating arachidonic acid metabolic network and the deactivation of PI3K/Akt in human breast cancer. Toxicol Appl Pharmacol 13: 261-265, 2013.

35. Babu RL, Naveen Kumar M, Patil RH, Devaraju KS, Ramesh GT and Sharma SC: Effect of estrogen and tamoxifen on the expression pattern of AP-1 factors in MCF-7 cells: role of c-jun, c-fos, and fra-1 in cell cycle regulation. Mol Cell Biochem 380: 143-151, 2013.

36. Hsieh CY, Tsai PC, Chu CL, Chang FR, Chang LS, Wu YC and Lin SR: Brazilein suppresses migration and invasion of MDA-MB-231 breast cancer cells. Chem Biol Interact 2: 105-115, 2013. 